\title{
Revenue Sharing in Airline Alliance Networks
}

\author{
Yuntong Wang (University of Windsor)
}

Working paper $16-05$

Working papers are in draft form. This working paper is distributed for purposes of comment and discussion only. It may not be reproduced without permission of the copyright holder. Copies of working papers are available from the author or at http://ideas.repec.org/s/wis/wpaper.html. 


\title{
Revenue Sharing in Airline Alliance Networks
}

\author{
Yuntong Wang* \\ Department of Economics \\ University of Windsor \\ Windsor, Ontario, Canada
}

March 23, 2016

*Tel: (519) 253-3000 ext.2382; Fax: (519) 973-7096; E-mail: yuntong@uwindsor.ca 


\begin{abstract}
This paper takes an axiomatic approach to the revenue sharing problem for an airline alliance network. We propose a simple sharing rule that allocates the revenue of each flight equally among the carriers of the flight. We show that it is the only rule satisfying the axioms of Separability, the Null Airline Property, and Equal Treatment of Equals. We show that the rule coincides with the Shapley value of the game associated with the problem. We provide two extensions of the rule, allowing it to depend on the lengths or the capacities of the flight legs. We also consider the maximum revenue problem for the airline alliance. We propose a simple Integer Linear Programming model. We examine its Owen set. Lastly, we provide an algorithm to compute both the optimal solution and the revenue sharing solution given by the simple sharing rule for the maximum revenue problem.
\end{abstract}

JEL classification: C71, D70

Keywords: Revenue sharing, Airline alliance, Network 


\section{Introduction}

An airline alliance is an arrangement between two or more airlines agreeing to cooperate and share their resources (e.g., legs). In doing so, they can expand their flights between more Origin and Destination (OD) pairs or increase their capacities and offer more flights on the existing network. In an airline alliance, each airline expects its own revenue share as large as possible. For a successful or stable alliance, a fair revenue sharing rule is essential.

There are two different approaches to the revenue sharing problem. ${ }^{1}$ In the cooperative game theory approach, a Linear Programming (LP) model is usually defined for the maximum expected revenue for the airline alliance. ${ }^{2}$ The maximum value of the LP problem for each coalition of the airlines generates a TU game, called the airline alliance revenue management game. The core of the game has been the main focus in the literature (Kimms and Çetiner, 2012).

In the noncooperative game theory approach, airlines maximize their own revenue independently. In Netessine and Shumsky (2005), horizontal and vertical competition have been considered separately. In the horizontal game, airlines are in direct competition on a given OD pair through parallel flights, and seats on different flights are substitutable. In the vertical game, each airline operates a different leg in a network of interconnected flights. Thus, seats on different legs are complementary. Netessine and Shumsky studied and compared the Nash equilibria of the two games, respectively.

Recently, Hu et al. (2013) combine the cooperative and the noncooperative approaches. In their model, airlines first agree on a revenue sharing rule and then implement the rule in a decentralized operation of their flight reservation system. Specifically, they consider a two-stage game. In the first stage, they use a cooperative game framework to model the output of the negotiation in which airlines decide a revenue sharing rule that they will use to split the revenue of interline and codeshare itineraries. Hu et al. require that the sharing rules have the core property. In the second stage of the game, they consider efficiency by modeling the operation of the alliance as a noncooperative game in a decentralized network. Each airline manages its

\footnotetext{
${ }^{1}$ Revenue sharing problem is often treated as a part of the more general so-called Revenue Management Problem in the airline industry. See Talluri and Ryzin (2004).

${ }^{2}$ Because demands for flights are uncertain before the departure of the flights, they have to be estimated.
} 
own reservation system so as to maximize its expected revenue.

While the cooperative game approach to the revenue sharing problem is very useful, it has two drawbacks. First, it relies on the definition of the game associated with the problem. Second, it ignores the information about the network structure of the problem. In this paper, we take an axiomatic approach to the airline alliance revenue sharing problem. One of the advantages of the axiomatic approach is that the revenue sharing rules can be based on certain desirable properties that are directly related to the network structure of the airline alliance and are independent of any notion of game theory.

We consider a network model of the airline alliance. For simplicity, in our model we assume that the demands for flights are perfectly elastic and any supply of flights can be sold. ${ }^{3}$ Specifically, we model the problem as a revenue sharing problem on a tree network, in which various paths between pairs of nodes (i.e., OD pairs) are flights that generate revenues. Each airline owns a part (a subset of legs) of the network and the whole network is jointly shared by all airlines in the alliance.

We first propose a simple sharing rule that allocates the revenue of each flight equally among the airlines that are the carriers of the flight. We provide a characterization of the rule by the axioms of Separability, the Null Airline Property, and Equal Treatment of Equals (Theorem 1). Separability is similar to the Additivity axiom in the cost sharing literature (Moulin, 2002) and it says that the allocation of the total revenue of all the flights can be decomposed into problems of allocating the revenue of each individual flight. The Null Airline Property is similar to the Dummy axiom in cost sharing and can be considered as an equity axiom (see also Moulin, 2002). The Equal Treatment of Equals treats all carriers equally in a flight. We also show that the rule coincides with the Shapley value of the game associated with the problem (Proposition 1).

To take into accounts the differences in the lengths or capacities of the legs with different airlines, we consider two extensions of the model, one in which each leg is associated with a length and one with a capacity. We then replace the Equal Treatment of Equals axiom with two different axioms, respectively. Accordingly, we provide two extensions of the rule. The first is related to the lengths of the legs and the second to their capacities. We

\footnotetext{
${ }^{3}$ This assumption allows us to focus on the revenue sharing problem.
} 
provide their characterizations (Theorems 2 and 3), respectively.

We also consider the problem of sharing the maximum revenue. Traditionally, the focus of the airline alliance is to maximize the total revenue they can jointly achieve. The game defined from the maximum revenue problem for each coalition of the airlines can be considered as conditions on how the total revenue of the alliance should be shared between the airlines. As mentioned before, to simplify the problem we assume that all flights that can be offered will be sold and thus generate revenue. ${ }^{4}$ The problem then can be simply modeled as an Integer Linear Programming (ILP) problem. Since there is no duality theory for an ILP problem and thus, we cannot define the Owen set (Owen, 1975) for the problem directly. ${ }^{5}$ We consider the LP Relaxation of the ILP and show that they have the same set of solutions (Lemma 1). Based on this result, we show that the core of the game is nonempty (Theorem 4). Then, the Owen set of the LP Relaxation problem can be defined and considered as a solution to the original maximum revenue sharing problem.

Lastly, we propose an algorithm that solves the maximum revenue problem and at the same time implements the simple revenue sharing rule introduced in the beginning.

\subsection{Relation to the Literature}

In the economic literature, revenue sharing problem in networks has not been widely studied except Ginsburgh and Zang (2004), Bergantiños and MorenoTernero (2015). On the other hand, there is a large related OR literature on the revenue management in the airline alliances (Talluri and van Ryzin, 2004). In the latter, however, revenue sharing is considered as secondary in the revenue management problem.

In contrast, cost allocation in networks has been widely studied (see Sharkey (1995) for a survey). Here we just mention a few. Littlechild and

\footnotetext{
${ }^{4}$ We essentially just consider the capacity constraints and remove the demand constraints on the feasible flights in the maximum revenue problem.

${ }^{5}$ In the OR literature, the maximum revenue problem is simply considered as a LP problem by ignoring the integer constraints, implicitly assuming that all the solutions of the LP problem are integer-valued. Then, the Owen set can be defined by the dual problem. Allocations in the Owen set are often used as revenue sharing rules. See Kimms and Çetiner, 2012.
} 
Owen (1973) consider the airport games, Bergantiños and Vidal-Puga (2007) on the minimum cost spanning tree games, Ni and Wang (2007) on the cost sharing problem of a linear polluted river, Bogomolnaia et al. (2010) on the cost sharing problem of a capacity network, and Moulin and Laigret (2011) on the cost sharing problem with multiple goods.

\subsection{Organization of the Paper}

We define a revenue sharing model for the airline alliance network and introduce a simple sharing rule in Section 2. In Section 3, we provide a characterization of the simple rule. In Section 4, we show that the simple rule coincides with the Shapley value of the game associated with the problem. In Section 5, we show that the simple rule also satisfies two additional properties: the merging-proofness and the core property. In Section 6, we provide two extensions of the simple rule. In Section 7 , we consider the maximum revenue sharing problem. In Section 8, we discuss an algorithmic approach to the maximum revenue sharing problem. Section 9 concludes the paper.

\section{The Model}

Let $\mathcal{A}=\{1,2,3, \ldots\}$ be the set of all possible airlines. An airline network is a graph $g=(N, E)$, where $N=\{1, \ldots, n\}$ is a finite set of nodes (e.g., airports) and $E$ is a collection of links (called legs) on $N$. We further assume that $g$ is a tree graph. Let $A \subset \mathcal{A}$ be a finite set of airlines. Assume that each airline owns a certain number of links in $E$ and each link in $E$ is owned by only one airline in $A$. In other words, for each $a \in A$, there is a set $E_{a} \subseteq E$ and $\left\{E_{a}\right\}_{a \in A}$ is a partition of $E$. A flight $f_{i j}$ between $i \in N$ and $j \in N(j \neq i)$ is a path in $g$, i.e., a set of links $\left\{\left(n_{l-1}, n_{l}\right)\right\}_{l \in\{2, \ldots, d\}}$ such that $i=n_{1}$ and $j=n_{d} .{ }^{6}$ Denote $f_{i j}=\left\{\left(n_{l-1}, n_{l}\right)\right\}_{l \in\{2, \ldots, d\}}$. Let $F$ be the set of all possible flights on $g$. Given a flight $f_{i j} \in F$, we say $f_{i j}$ is shared by the airline alliance $A\left(f_{i j}\right) \subseteq A$ if and only if an airline $a \in A\left(f_{i j}\right)$ owns a link (or links) in $f_{i j}$. Note that for each flight $f_{i j}$, there is a unique $A\left(f_{i j}\right)$. A flight profile, $f$, is a subset of $F$, i.e., $f \subseteq F$. Suppose that each flight $f_{i j}$ generates a revenue $r\left(f_{i j}\right) \in R_{+}$. The total revenue of $f$, is thus, $\sum_{f_{i j} \in f} r\left(f_{i j}\right)$.

\footnotetext{
${ }^{6}$ Since $g$ is a tree, there is a unique path between any pair of nodes.
} 
A revenue sharing problem is a tuple $(A, g, f, r)$. Since $A$ is given, we denote a problem by $(g, f, r) .^{7}$ Given a problem $(g, f, r)$, a solution is a vector $x \in R_{+}^{A}$ such that

$$
\sum_{a \in A} x_{a}=\sum_{f_{i j} \in f} r\left(f_{i j}\right)
$$

A revenue sharing rule is a mapping $x$ that assigns to each problem $(g, f, r)$ a solution $x(g, f, r)$.

We first consider the following rule that allocates the revenue of each flight equally to the airlines that are the carriers of the flight. Formally,

$$
x_{a}(g, f, r)=\sum_{f_{i j} \in f: a \in A\left(f_{i j}\right)} \frac{r\left(f_{i j}\right)}{\left|A\left(f_{i j}\right)\right|}, a \in A,
$$

where $\left|A\left(f_{i j}\right)\right|$ is the number of airlines in the set $A\left(f_{i j}\right)$.

Example 1. In Figure 1 below, there are three airlines $a, b$, and $c$, and six airports. Suppose $f=\left\{f_{16}, f_{34}, f_{25}, f_{26}\right\}$. The total revenue of $f$ is thus

$$
r\left(f_{16}\right)+r\left(f_{34}\right)+r\left(f_{25}\right)+r\left(f_{26}\right),
$$

which is to be shared between the three airlines. Note that $A\left(f_{16}\right)=\{a, b, c\}, A\left(f_{34}\right)=$ $\{b\}, A\left(f_{25}\right)=\{b\}, A\left(f_{26}\right)=\{b, c\}$.

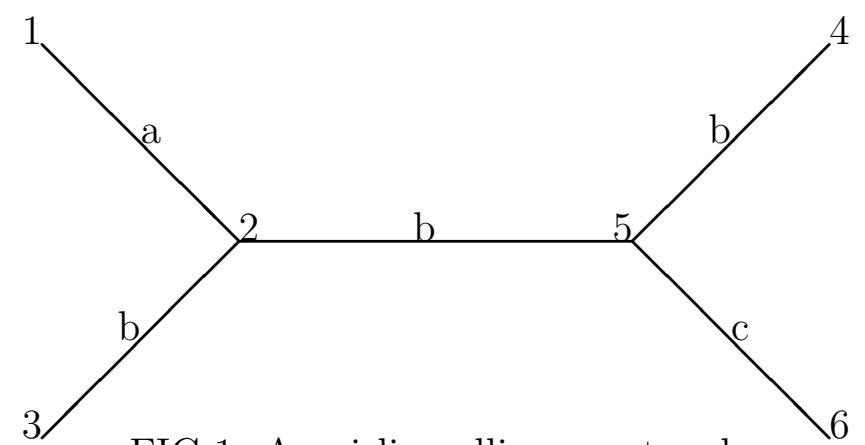

FIG.1. An airline alliance network

By the revenue sharing rule (1), we have

\footnotetext{
${ }^{7}$ In Section 5 , when we discuss airline merging, we will include $A$ again.
} 


$$
\begin{gathered}
x_{a}=\frac{1}{3} r\left(f_{16}\right), \\
x_{b}=r\left(f_{25}\right)+r\left(f_{34}\right)+\frac{1}{3} r\left(f_{16}\right)+\frac{1}{2} r\left(f_{26}\right), \\
x_{c}=\frac{1}{3} r\left(f_{16}\right)+\frac{1}{2} r\left(f_{26}\right) .
\end{gathered}
$$

\section{The Characterization}

For a characterization of (1), we use the following axioms.

Separability: Let $\left(g, f^{1}, r\right)$ and $\left(g, f^{2}, r\right)$ be two problems such that $f^{1} \cap f^{2}=\emptyset$. Then

$$
x_{a}\left(g, f^{1} \cup f^{2}, r\right)=x_{a}\left(g, f^{1}, r\right)+x_{a}\left(g, f^{2}, r\right), a \in A .
$$

Separability is similar to the Additivity axiom in the cost sharing literature (Moulin, 2002). It allows us to decompose the revenue sharing for all the flights into sharing each individual flight in the profile. This implies that there is no cross-subsidization between flights.

The Null Airline Property: Let $(g, f, r)$ be a problem and $a \in A$. If $a \notin \cup_{f_{i j} \in f} A\left(f_{i j}\right)$, then $x_{a}(g, f, r)=0$.

This axiom says that if an airline is not a carrier in any of the flights in a given profile, the airline should not receive any revenue. This axiom is similar to the Dummy axiom in the cost sharing literature (see also Moulin, 2002).

Equal Treatment of Equals: Let $(g, f, r)$ be a problem and $a, a^{\prime} \in A$ be two airlines. If, for every $f_{i j} \in f, a \in A\left(f_{i j}\right)$ implies $a^{\prime} \in A\left(f_{i j}\right)$, then $x_{a}(g, f, r)=x_{a^{\prime}}(g, f, r)$.

The Equal Treatment of Equals is a symmetry axiom, which treats all airlines in the same flight equally. 
Theorem 1 The rule defined in (1) is the only rule that satisfies the axioms of Separability, Null Airline Property, and Equal Treatment of Equals.

Proof. It is easy to check that the rule (1) satisfies the above three axioms.

Now suppose that a sharing rule $\varphi$ satisfies the three axioms. We will show that $\varphi$ must be given by (1). Fix an arbitrary $f$. Note that we can write $f=\cup_{f_{i j} \in f}\left\{f_{i j}\right\}$. Now consider the flight profile $\left\{f_{i j}\right\}$. By the Null Airline Property and the Equal Treatment of Equals axioms, we have $\varphi_{a}\left(g,\left\{f_{i j}\right\}, r\right)=0$ for all $a \in A \backslash A\left(f_{i j}\right)$, and $\varphi_{a}\left(g,\left\{f_{i j}\right\}, r\right)=r\left(f_{i j}\right) /\left|A\left(f_{i j}\right)\right|$ for all $a \in A\left(f_{i j}\right)$.

Then by Separability, we have

$$
\begin{aligned}
\varphi_{a}(g, f, r) & =\varphi_{a}\left(g, \cup_{f_{i j} \in f}\left\{f_{i j}\right\}, r\right) \\
& =\sum_{f_{i j} \in f} \varphi_{a}\left(g,\left\{f_{i j}\right\}, r\right) \\
& =\sum_{f_{i j} \in f: a \notin A\left(f_{i j}\right)} 0+\sum_{f_{i j} \in f: a \in A\left(f_{i j}\right)} \frac{r\left(f_{i j}\right)}{\left|A\left(f_{i j}\right)\right|} \\
& =\sum_{f_{i j} \in f: a \in A\left(f_{i j}\right)} \frac{r\left(f_{i j}\right)}{\left|A\left(f_{i j}\right)\right|}
\end{aligned}
$$

for all $a \in A$.

This completes the proof of the theorem.

Q.E.D.

\section{The Shapley Value}

We now show that the rule (1) coincides with the Shapley value (Shapley, 1953) of the following revenue game $r(\cdot)$ that is associated with the problem $(g, f, r)$ :

$$
r(S)=\sum_{\left\{f_{i j} \in f \mid A\left(f_{i j}\right) \subseteq S\right\}} r\left(f_{i j}\right), \quad S \subseteq A .
$$

The Shapley value of the game $r(\cdot)$ is given by

$$
\phi_{a}(r)=\sum_{S \subseteq A: a \in S} \frac{(|S|-1) !(|A|-|S|) !}{|A| !}[r(S)-r(S \backslash\{a\})], a \in A .
$$


Proposition 1 The Shapley value of the revenue game $r(\cdot)$ defined in (2) coincides with the rule (1).

Proof. Fix $a \in A$. Consider $f_{i j} \in f$. Define the following game:

$$
r^{i j}(S)=\left\{\begin{array}{ll}
r\left(f_{i j}\right) & \text { if } S \supseteq A\left(f_{i j}\right) \\
0 & \text { otherwise, }
\end{array} \quad S \subseteq A .\right.
$$

Clearly, all airlines in $A \backslash A\left(f_{i j}\right)$ are dummy in the game $r^{i j}$ and all airlines in $A\left(f_{i j}\right)$ are symmetric. Thus the Shapley value of the game $r^{i j}$ is given by

$$
\phi_{a^{\prime}}\left(r^{i j}\right)=\left\{\begin{array}{c}
\frac{r\left(f_{i j}\right)}{\left|A\left(f_{i j}\right)\right|}, a^{\prime} \in A\left(f_{i j}\right) \\
0, \text { otherwise }
\end{array}\right.
$$

for all $a^{\prime} \in A$.

Since the game $r(S)=\sum_{f_{i j} \in f} r^{i j}(S)$, the additivity of the Shapley value implies

$$
\begin{aligned}
\phi_{a}(r) & =\sum_{f_{i j} \in f} \phi_{a}\left(r^{i j}\right) \\
& =\sum_{f_{i j} \in f: a \in A\left(f_{i j}\right)} \frac{r\left(f_{i j}\right)}{\left|A\left(f_{i j}\right)\right|} .
\end{aligned}
$$

This completes the proof of the proposition.

Q.E.D.

\section{The Merging-Proofness and the Core Prop- erty}

Airline merging is an important issue in the airline industry (Morrison and Winston, 1986). However, airline alliance can be beneficial for customers as long as they do not collude on pricing. We show below that, from the revenue sharing aspect, under the revenue sharing rule (1) airlines would have no incentive to merge.

Merging-proofness: Let $(A, g, f, r)$ and $(\bar{A}, g, f, r)$ be two problems where $a_{1}, a_{2} \in A, \bar{A}=A \backslash\left\{a_{1}, a_{2}\right\} \cup\{a\}$, and $E_{a}=E_{a_{1}} \cup E_{a_{2}}$ in $(\bar{A}, g, f, r)$. That is, in $(\bar{A}, g, f, r), a_{1}, a_{2}$ are replaced by $a$ in all flights that involve $a_{1}$ or 
$a_{2}$ (or both). We say that the revenue sharing rule $x$ satisfies the mergingproofness if

$$
x_{a}(\bar{A}, g, f, r) \leq x_{a_{1}}(A, g, f, r)+x_{a_{2}}(A, g, f, r) .
$$

We show below that the rule (1) satisfies the merging-proofness. Indeed,

$$
\begin{array}{r}
x_{a}(\bar{A}, g, f, r)=\sum_{f_{i j} \in f: a \in \bar{A}\left(f_{i j}\right)} \frac{r\left(f_{i j}\right)}{\left|\bar{A}\left(f_{i j}\right)\right|} \\
=\sum_{f_{i j} \in f: a_{1} \in A\left(f_{i j}\right), a_{2} \notin A\left(f_{i j}\right)} \frac{r\left(f_{i j}\right)}{\left|A\left(f_{i j}\right)\right|}+\sum_{f_{i j} \in f: a_{2} \in A\left(f_{i j}\right), a_{1} \notin A\left(f_{i j}\right)} \frac{r\left(f_{i j}\right)}{\left|A\left(f_{i j}\right)\right|} \\
+\sum_{f_{i j} \in f: a_{1} \in A\left(f_{i j}\right), a_{2} \notin A\left(f_{i j}\right)} \frac{r\left(f_{i j}\right)}{\left|A\left(f_{i j}\right)\right|}+\sum_{f_{i j} \in f: a_{1} \in A\left(f_{i j}\right), a_{2} \in A\left(f_{i j}\right)} \frac{\sum_{\mid A\left(f_{i j}\right), a_{1} \notin A\left(f_{i j}\right)} \frac{r\left(f_{i j}\right)}{\left|A\left(f_{i j}\right)\right|}}{+2} \sum_{f_{i j} \in f: a_{1} \in A\left(f_{i j}\right), a_{2} \in A\left(f_{i j}\right)} \frac{r\left(f_{i j}\right)}{\left|A\left(f_{i j}\right)\right|} \\
=x_{a_{1}(A, g, f, r)+x_{a_{2}}(A, g, f, r) .}
\end{array}
$$

(We assume that $\left|A\left(f_{i j}\right)\right| \geq 2, \forall f_{i j} \in f$.)

While airline alliances are beneficial to customers, they shall be beneficial to airlines as well. A requirement for a revenue sharing rule to be acceptable by the alliance is the core property. Without it, some airlines may not want to join the alliance or may want to leave an alliance they have joined.

In the following, we show that the simple sharing rule has the core property. In fact, we show first that the revenue game defined in (2) is convex. Therefore, by Proposition 1, the allocation given by the rule (1) is in the core of the game (2) (Shapley, 1971).

In fact, for any $S, T \subseteq A$ and $S \subset T$ and $a \notin T$, we have

$$
\begin{aligned}
\sum_{\left\{f_{i j} \in f \mid A\left(f_{i j}\right) \subseteq S \cup\{a\}\right\}} r\left(f_{i j}\right)-\sum_{\left\{f_{i j} \in f \mid A\left(f_{i j}\right) \subseteq S\right\}} r\left(f_{i j}\right) & =\sum_{\left\{f_{i j} \in f \mid a \in A\left(f_{i j}\right), A\left(f_{i j}\right) \backslash\{a\} \subseteq S\right\}} r\left(f_{i j}\right) \\
=\sum_{\left\{f_{i j} \in f \mid A\left(f_{i j}\right) \subseteq T \cup\{a\}\right\}} r\left(f_{i j}\right)-\sum_{\left\{f_{i j} \in f \mid A\left(f_{i j}\right) \subseteq T\right\}} r\left(f_{i j}\right), &
\end{aligned}
$$

which shows that the game (2) is convex. 


\section{Two Extensions}

In an airline network, it is unlikely that all legs in a flight have equal distances. Generally speaking, longer legs generate higher revenues than shorter ones. Also, different legs may have different flight capacities. Therefore, it is not always practical to treat all legs equally.

In the following, we consider two different models from the model in Section 2. Specifically, we make two additional assumptions on the airline alliance network. First, we assume that in the network each link is associated with a distance. Formally, let $g^{d} \equiv(g, d)$, where $d: E \rightarrow R_{+}$is a function that assigns to each link $e \in E$ a distance $d(e) \in R_{+}$. Now, a revenue sharing problem is a tuple $\left(g^{d}, f, r\right)$.

To treat longer legs differently from short ones, we introduce the following axiom.

Distance Proportionality: For any individual flight $f_{i j} \in f$, if $a \in$ $A\left(f_{i j}\right)$ and $d_{a}\left(f_{i j}\right) \in R_{+}$is the total distance of the legs by airline $a$ in the flight $f_{i j}$, i.e., $d_{a}\left(f_{i j}\right)=\sum_{e_{a} \in E_{a} \cap f_{i j}} d\left(e_{a}\right)$, then airline $a$ shares $d_{a}\left(f_{i j}\right) / \sum_{a^{\prime} \in A\left(f_{i j}\right)} d_{a^{\prime}}\left(f_{i j}\right)$ proportion of the revenue $r\left(f_{i j}\right)$.

The distance-weighted rule is defined by

$$
x_{a}\left(g^{d}, f, r\right)=\sum_{f_{i j} \in f: a \in A\left(f_{i j}\right)} \frac{d_{a}\left(f_{i j}\right)}{\sum_{a^{\prime} \in A\left(f_{i j}\right)} d_{a^{\prime}}\left(f_{i j}\right)} r\left(f_{i j}\right), a \in A .
$$

We have the following characterization of (7).

Theorem 2 The distance-weighted rule (7) is the only rule satisfying Separability, the Null Airline Property, and Distance Proportionality.

Proof. It is easy to check that the rule (7) satisfies the axioms of Separability, Null Airline Property, and Distance Proportionality.

Now suppose that a sharing rule $\varphi$ satisfies the three axioms. We show that it must be given by (7). Fix an arbitrary $f$. Note that we can write $f=\cup_{f_{i j} \in f}\left\{f_{i j}\right\}$. Now consider the flight profile $\left\{f_{i j}\right\}$. By the Null Airline Property and Distance Proportionality, we have $\varphi_{a}\left(g^{d},\left\{f_{i j}\right\}, r\right)=0$ for all $a \in A \backslash A\left(f_{i j}\right)$, and $\varphi_{a}\left(g^{d},\left\{f_{i j}\right\}, r\right)=\left(d_{a}\left(f_{i j}\right) / \sum_{a^{\prime} \in A\left(f_{i j}\right)} d_{a^{\prime}}\left(f_{i j}\right)\right) r\left(f_{i j}\right)$ for all $a \in A\left(f_{i j}\right)$. 
By Separability, we have

$$
\begin{aligned}
\varphi_{a}\left(g^{d}, f, r\right) & =\varphi_{a}\left(g^{d}, \cup_{f_{i j} \in f}\left\{f_{i j}\right\}, r\right) \\
& =\sum_{f_{i j} \in f} \varphi_{a}\left(g^{d},\left\{f_{i j}\right\}, r\right) \\
& =\sum_{f_{i j} \in f: a \notin A\left(f_{i j}\right)} 0+\sum_{f_{i j} \in f: a \in A\left(f_{i j}\right)} \frac{d_{a}\left(f_{i j}\right)}{\sum_{a^{\prime} \in A\left(f_{i j}\right)} d_{a^{\prime}}\left(f_{i j}\right)} r\left(f_{i j}\right) \\
& =\sum_{f_{i j} \in f: a \in A\left(f_{i j}\right)} \frac{d_{a}\left(f_{i j}\right)}{\sum_{a^{\prime} \in A\left(f_{i j}\right)} d_{a^{\prime}}\left(f_{i j}\right)} r\left(f_{i j}\right),
\end{aligned}
$$

for all $a \in A$.

This completes the proof of the theorem.

Q.E.D.

In the second extension of the model, we assume that each link of the network has a capacity constraint. ${ }^{8}$ Let $g^{c} \equiv(g, c)$, where $c: E \rightarrow I=\{1,2, \ldots\}$ is a function that assigns to each link (leg) a capacity. Now a problem is a tuple $\left(g^{c}, f, r\right)$, where $f$ is a feasible flight profile for $g^{c}$ defined as follows.

Call a flight profile, $f \subseteq F$, feasible for $\left(g^{c}, r\right)$ if

$$
\sum_{\left\{f_{i j} \in f \mid e_{a} \in f_{i j}\right\}} f_{i j} \leq c\left(e_{a}\right), e_{a} \in E_{a}, a \in A,
$$

where $f_{i j}$, with a slight abuse of notation, also represents the number of seats in the flight $f_{i j}$.

Note that now the total revenue of the flight profile $f$, is $\sum_{f_{i j} \in f} r\left(f_{i j}\right) f_{i j}$, where $r\left(f_{i j}\right)$ is considered as the unit revenue of the flight $f_{i j}$.

The capacity-weighted rule is defined by

$$
x_{a}\left(g^{c}, f, r\right)=\sum_{f_{i j} \in f: a \in A\left(f_{i j}\right)} \frac{C\left(a, f_{i j}\right)}{\sum_{a^{\prime} \in A\left(f_{i j}\right)} C\left(a^{\prime}, f_{i j}\right)} r\left(f_{i j}\right) f_{i j}, a \in A,
$$

${ }^{8} \mathrm{It}$ is possible to define other rules that depend on both the distance and the capacity. We omit these possible extensions. 
where $C\left(a, f_{i j}\right)=\sum_{e_{a} \in E_{a} \cap f_{i j}} c\left(e_{a}\right)$ is the total capacity of airline $a$ for the flight $f_{i j}$.

Accordingly, we introduce the following axiom.

Capacity Proportionality: For any flight $f_{i j} \in f$, if $a \in A\left(f_{i j}\right)$ and $C\left(a, f_{i j}\right)$ is the total capacity of airline $a$ for the flight $f_{i j}$, then airline $a$ shares $C\left(a, f_{i j}\right) / \sum_{a^{\prime} \in A\left(f_{i j}\right)} C\left(a^{\prime}, f_{i j}\right)$ proportion of the revenue $r\left(f_{i j}\right) f_{i j}$.

We have the following characterization of (8).

Theorem 3 The capacity-weighted rule (8) is the only rule satisfying Separability, Null Airline Property, and Capacity Proportionality.

Proof. It is easy to see that the rule (8) satisfies the three axioms.

Now suppose that a sharing rule $\varphi$ satisfies the three axioms. We show that it must be given by (8). Fix an arbitrary $f$. Note that we can write $f=\cup_{f_{i j} \in f}\left\{f_{i j}\right\}$. Now consider the flight profile $\left\{f_{i j}\right\}$. By the Null Airline Property and Capacity Proportionality, we have $\varphi_{a}\left(g^{c},\left\{f_{i j}\right\}, r\right)=0$ for all $a \in A \backslash A\left(f_{i j}\right)$, and $\varphi_{a}\left(g^{c},\left\{f_{i j}\right\}, r\right)=\left(C\left(a, f_{i j}\right) / \sum_{a^{\prime} \in A\left(f_{i j}\right)} C\left(a^{\prime}, f_{i j}\right)\right) r\left(f_{i j}\right) f_{i j}$ for all $a \in A\left(f_{i j}\right)$.

By Separability, we have

$$
\begin{aligned}
\varphi_{a}\left(g^{c}, f, r\right) & =\varphi_{a}\left(g^{c}, \cup_{f_{i j} \in f}\left\{f_{i j}\right\}, r\right) \\
& =\sum_{f_{i j} \in f} \varphi_{a}\left(g^{c},\left\{f_{i j}\right\}, r\right) \\
& =\sum_{f_{i j} \in f: a \notin A\left(f_{i j}\right)} 0+\sum_{f_{i j} \in f: a \in A\left(f_{i j}\right)} \frac{C\left(a, f_{i j}\right)}{\sum_{a^{\prime} \in A\left(f_{i j}\right)} C\left(a^{\prime}, f_{i j}\right)} r\left(f_{i j}\right) f_{i j} \\
& =\sum_{f_{i j} \in f: a \in A\left(f_{i j}\right)} \frac{C\left(a, f_{i j}\right)}{\sum_{a^{\prime} \in A\left(f_{i j}\right)} C\left(a^{\prime}, f_{i j}\right)} r\left(f_{i j}\right) f_{i j}
\end{aligned}
$$

for all $a \in A$.

This completes the proof of the theorem.

Q.E.D. 


\section{Sharing the Maximum Revenue}

In the airline revenue management literature (e.g., de Boer et al., 2002), a Mathematical Programming (MP) problem is defined to maximize the airline's expected revenue from its supply of OD pairs, using the estimated demand distribution to define constraints on flights. Extending this airline revenue management problem from single airline to airline alliance is not straightforward. One of the issues is how the airlines should share the joint maximum revenue. For any airline alliance, airlines must first agree on a revenue sharing rule.

Consider a problem $\left(A, g^{c}, r\right)$ (see Section 6). Assume that $f^{*}$ is a revenue maximizing flight profile for $\left(A, g^{c}, r\right)$. Let $R\left(f^{*}\right)=\sum_{f_{i j} \in f^{*}} r\left(f_{i j}\right) f_{i j}$ be the maximum revenue. How should $R\left(f^{*}\right)$ be shared among the airlines in $A$ ?

We first give a brief review of the traditional game theoretic approach. Then we will introduce our algorithmic approach, which is related to our axiomatic approach to the revenue sharing problem.

The game theoretic approach begins with the following definition. The maximum revenue game of the problem $\left(A, g^{c}, r\right)$ is defined as follows: for any $S \subseteq A$,

$$
\begin{aligned}
R(S)= & \max \sum_{\left\{f_{i j} \in F \mid A\left(f_{i j}\right) \subseteq S\right\}} r\left(f_{i j}\right) f_{i j} \\
\text { s.t. } & \sum_{\left\{f_{i j} \mid e_{a} \in f_{i j}\right\}} f_{i j} \leq c\left(e_{a}\right), e_{a} \in E_{a}, a \in S, \\
& f_{i j} \geq 0 \text { integer }, i<j, i, j \in N .
\end{aligned}
$$

Note that problem (9) is an ILP problem. In the lemma below, we show that for (9), it is equivalent to solve its LP Relaxation.

Lemma 1 The Integer Linear Programming problem defined in (9) can be solved by solving its LP Relaxation.

Proof. Without loss of generality, assume that all $r\left(f_{i j}\right)$ are different. Note that all $c\left(e_{a}\right)$ are integers. Suppose that $f^{*}=\left\{f_{i j}^{*}\right\}_{i<j}$ is an optimal solution for the LP Relaxation of (9). We shall show that all $f_{i j}^{*}$ are integers. Therefore, $f^{*}$ is also an optimal solution of $(9)$. We prove this by contradiction. 
For simplicity, suppose that there are only two $f_{i_{1} j_{1}}^{*}$ and $f_{i_{2} j_{2}}^{*}$ that are not integers. ${ }^{9}$ Assume that $r\left(f_{i_{1} j_{1}}^{*}\right)>r\left(f_{i_{2} j_{2}}^{*}\right)$.

By the integer capacity constraints, if any constraint for $f^{*}$ is satisfied with equality and at the same time, contains of one of the two non-integers, $f_{i_{1} j_{1}}^{*}$ and $f_{i_{2} j_{2}}^{*}$, it must contain both. Suppose one of these constraints is in the following form:

$$
f_{i_{1} j_{1}}^{*}+f_{i_{2} j_{2}}^{*}+\ldots=c\left(e_{a}^{*}\right) .
$$

Then we can always find a small $\epsilon>0$ and define a new feasible profile $f^{* *}$ in which,

$$
\begin{gathered}
f_{i_{1} j_{1}}^{* *}=f_{i_{1} j_{1}}^{*}+\epsilon, \\
f_{i_{2} j_{2}}^{* *}=f_{i_{2} j_{2}}^{*}-\epsilon, \\
f_{i j}^{* *}=f_{i j}^{*}, \text { all others. }
\end{gathered}
$$

Note that for any non-binding constraint that contains only one of the non-integers $f_{i_{1} j_{1}}^{*}$ and $f_{i_{2} j_{2}}^{*}$, we can make $\epsilon$ small enough so that it would still be non-binding. It is easy to see that the total revenue at $f^{* *}$ would be higher than that of $f^{*}$. This contradicts the assumption that $f^{*}$ is optimal.

This completes the proof of the Lemma.

Q.E.D.

Now we are ready to prove the following theorem.

Theorem 4 The game (9) is balanced and any solution to the dual problem of $R(A)$ is a core allocation.

Proof. Consider the following problem

$$
\begin{aligned}
R(A)= & \max \sum_{\left\{f_{i j} \in F \mid A\left(f_{i j}\right) \subseteq A\right\}} r\left(f_{i j}\right) f_{i j} \\
\text { s.t. } & \sum f_{i j} \leq c\left(e_{a}\right), e_{a} \in E_{a}, a \in A, \\
& f_{i j} \geq 0 \text { integer, } i<j, i, j \in N .
\end{aligned}
$$

By Lemma 1, the above problem and its LP Relaxation below have the same set of solutions.

$$
\bar{R}(A)=\max \sum_{\left\{f_{i j} \in F \mid A\left(f_{i j}\right) \subseteq A\right\}} r\left(f_{i j}\right) f_{i j}
$$

\footnotetext{
${ }^{9}$ For other cases, the proof is similar and is omitted.
} 


$$
\begin{array}{ll}
\text { s.t. } & \sum_{\left\{f_{i j} \mid e_{a} \in f_{i j}\right\}} f_{i j} \leq c\left(e_{a}\right), e_{a} \in E_{a}, a \in A, \\
& f_{i j} \geq 0, i<j, i, j \in N .
\end{array}
$$

Now we can consider the latter's dual problem:

$$
\begin{aligned}
\bar{R}^{*}(A)= & \min \sum_{a \in A} \sum_{e_{a} \in E_{a}} \lambda\left(e_{a}\right) c\left(e_{a}\right) \\
\text { s.t. } & \sum_{a \in A\left(f_{i j}\right)} \sum_{e_{a} \in E_{a} \cap f_{i j}} \lambda\left(e_{a}\right) \geq r\left(f_{i j}\right), \\
& \lambda\left(e_{a}\right) \geq 0, e_{a} \in E_{a}, a \in A .
\end{aligned}
$$

We show below that any solution to $(13),\left(\lambda\left(e_{a}\right)\right)_{e_{a} \in E_{a}, a \in A}$, would induce a core allocation to the game $R$ :

$$
\left(\sum_{e_{a} \in E_{a}} \lambda\left(e_{a}\right) c\left(e_{a}\right)\right)_{a \in A}
$$

Indeed, for any $S \subseteq A$, it is easy to see that the solution $\left(\lambda\left(e_{a}\right)\right)_{e_{a} \in E_{a}, a \in A}$ restricted to $S$ is also a feasible solution to the dual of the LP Relaxation of $R(S)$. Specifically, let

$$
\begin{aligned}
\bar{R}(S)= & \max \sum_{\left\{f_{i j} \in F \mid A\left(f_{i j}\right) \subseteq S\right\}} r\left(f_{i j}\right) f_{i j} \\
\text { s.t. } & \left.\sum f_{i j} \leq c\left(e_{a}\right), e_{a} \in E_{a} \in f_{i j}\right\} \\
& f_{i j} \geq 0, i<j, i, j \in N .
\end{aligned}
$$

The dual of $\bar{R}(S)$ is then

$$
\begin{aligned}
\bar{R}^{*}(S)= & \min \sum_{a \in S} \sum_{e_{a} \in E_{a}} \lambda\left(e_{a}\right) c\left(e_{a}\right) \\
\text { s.t. } & \sum_{a \in A\left(f_{i j}\right)} \sum_{e_{a} \in E_{a} \cap f_{i j}} \lambda\left(e_{a}\right) \geq r\left(f_{i j}\right), \\
& \lambda\left(e_{a}\right) \geq 0, e_{a} \in E_{a}, a \in S .
\end{aligned}
$$

It is easy to see that

$$
\sum_{a \in S} \sum_{e_{a} \in E_{a}} \lambda\left(e_{a}\right) c\left(e_{a}\right) \geq \bar{R}^{*}(S)=\bar{R}(S)=R(S), \forall S \subseteq A .
$$


The last equality is from Lemma 1.

This proves that the game (9) is balanced and a solution to the dual problem (13) is a core allocation to the game. This completes the proof of the theorem.

Q.E.D.

Since the core of the game (9) is usually a set of allocations, the core solution to the problem is not unique. Nevertheless, we can define the following solution concept. Given a problem, $\left(A, g^{c}, r\right)$. Consider the dual Linear Programming problem of the maximum revenue problem (12), namely, the problem (13). Let $\left(\lambda\left(e_{a}\right)\right)_{e_{a} \in E_{a}, a \in A}$ be one of its solutions. Define the following Owen value (Owen, 1975): ${ }^{10}$

$$
x_{a}^{O}\left(A, g^{c}, r\right)=\sum_{e_{a} \in E_{a}} \lambda\left(e_{a}\right) c\left(e_{a}\right), a \in A .
$$

By Theorem 4, the above solution gives a core allocation.

Remark: The duality approach induces a (shadow) price for each unit of the capacity on each leg. Since capacities are integer values, we shall be aware of the issue that the optimal value can be very sensitive to the change in capacity. Therefore, we shall be caution when we use the Owen value for revenue sharing.

Example 2. Consider the following airline network

$$
1 \stackrel{\mathrm{a}}{c_{12}=10^{2}} c_{23}=20^{3} c_{34}=10^{4}
$$

FIG.2. A linear airline alliance network

\footnotetext{
${ }^{10}$ The Owen set is the set of all the Owen values.
} 
Suppose that the revenue function is given as follows:

\begin{tabular}{|c|cccccc|}
\hline$f$ & $f_{12}$ & $f_{13}$ & $f_{14}$ & $f_{23}$ & $f_{24}$ & $f_{34}$ \\
\hline$r$ & 500 & 1,500 & 2,000 & 800 & 1,800 & 400 \\
\hline
\end{tabular}

The maximum revenue problem $R(A)$ is the following

$$
\begin{array}{ll} 
& \max 500 f_{12}+1,500 f_{13}+2,000 f_{14}+800 f_{23}+1,800 f_{24}+400 f_{34} \\
\text { s.t. } & f_{12}+f_{13}+f_{14} \leq 10 \\
& f_{13}+f_{23}+f_{14}+f_{24} \leq 20 \\
& f_{14}+f_{24}+f_{34} \leq 10 \\
& f_{i j} \geq 0, i<j, i, j=1,2,3
\end{array}
$$

Recall that $f_{i j}$ represents the number of seats.

It is easy to see that the optimal solution is $f_{13}^{*}=10, f_{24}^{*}=10$ and the maximum revenue is $15,000+18,000=33,000$.

Now consider the dual problem $R^{*}(A)$ :

$$
\begin{array}{ll} 
& \min 10 \lambda\left(c_{12}\right)+20 \lambda\left(c_{23}\right)+10 \lambda\left(c_{34}\right) \\
\text { s.t. } & \lambda\left(c_{12}\right) \geq 500, \\
& \lambda\left(c_{12}\right)+\lambda\left(c_{23}\right) \geq 1,500, \\
& \lambda\left(c_{12}\right)+\lambda\left(c_{23}\right)+\lambda\left(c_{34}\right) \geq 2,000, \\
& \lambda\left(c_{23}\right)+\lambda\left(c_{34}\right) \geq 1,800, \\
& \lambda\left(c_{23}\right) \geq 800, \\
& \lambda\left(c_{34}\right) \geq 400, \\
& \lambda\left(c_{12}\right), \lambda\left(c_{23}\right), \lambda\left(c_{34}\right) \geq 0 .
\end{array}
$$

The optimal solution is

$$
\lambda\left(c_{12}\right)=500, \lambda\left(c_{23}\right)=1,000, \lambda\left(c_{34}\right)=800 .
$$

According to the Owen solution (16), we have

$$
x_{a}^{O}=5,000, x_{b}^{O}=20,000, x_{c}^{O}=8,000 .
$$


It is easy to check that the above allocation is in the core of the game given below:

$$
\begin{gathered}
R(a)=5,000, R(b)=16,000, R(c)=4,000, \\
R(a, b)=23,000, R(b, c)=26,000, R(a, c)=9,000, \\
R(a, b, c)=33,000 .
\end{gathered}
$$

\section{An Algorithmic Approach}

The maximum revenue problem (11) is an Integer Linear Programming Problem. By Lemma 1, we can solve its LP Relaxation (12) by applying the Simplex Method (Dantzig, 1963). Since revenue sharing is also part of the problem, in the following we propose an algorithm based on the Simplex Method that solves both the maximum revenue problem and the revenue sharing problem. Moreover, the revenue sharing solution is given by the simple sharing rule (1).

We use a form of the Simplex Algorithm in Garfinkel and Nemhauser (1972, pp.30-31).

Algorithm: ${ }^{11}$ Given a problem $\left(A, g^{c}, r\right)$. Consider the problem (11). Apply the following algorithm to its LP Relaxation (12).

Step 1: (Initialization.) Begin with the basic feasible solution (BFS): $f=$ $\left\{f_{e}\right\}_{e \in E}$. Assign the revenue of each flight to the corresponding airline of the leg. Go to Step 2.

Step 2: (Test for optimality.) If so, the current BFS is optimal and the corresponding allocation is the revenue sharing allocation. If not, go to Step 3 .

Step 3: (Choose an entering basic variable.) Select $f_{i j}^{e}$ to be a new basic variable by the rule of the Simplex method. Go to Step 4.

Step 4: (Choosing a departing basic variable.) Select $f_{i j}^{d}$ to become a non-basic variable by the rule of the Simplex method. Go to Step 5.

\footnotetext{
${ }^{11}$ We omit the detail on how to deal with the issues of degeneracy and cycling with the Simplex Algorithm. See Garfinkel and Nemhauser (1972).
} 
Step 5: (Pivoting.) Pivot at the $(e, d)$ entry in the Simplex tableau and divide the revenue $r\left(f_{i j}^{e}\right) f_{i j}^{e}$ equally between airlines in $A\left(f_{i j}^{e}\right)$ and reduce an equal amount of the total revenue $r\left(f_{i j}^{d}\right) f_{i j}^{d}$ from all the airlines in $A\left(f_{i j}^{d}\right)$. Also adjust the revenue allocation to the airlines associated with the other basic variables according to their changes due to the pivoting. After all these changes are made, then go to Step 2.

Consider again Example 2 in the last section. The algorithm would generate the optimal solution $f_{13}^{*}=10, f_{24}^{*}=10$ with the maximum revenue $15,000+18,000=33,000$. In the meantime, the algorithm produces the revenue shares for the three airlines that coincide with that by the rule (1). That is

$$
\begin{aligned}
& x_{a}=(1 / 2) \times 15,000=7,500, \\
& x_{b}=(1 / 2) \times 15,000+(1 / 2) \times 18,000=16,500, \\
& x_{c}=(1 / 2) \times 18,000=9,000 .
\end{aligned}
$$

We demonstrate below how the Simplex Algorithm implements the above solution.

First, it is easy to find an initial BFS. We simply take all the single-leg flights as a staring BFS. Rewrite the objective function with the non-basic variables

$$
R=25,000+200 f_{13}+300 f_{14}+600 f_{24} .
$$

The $\operatorname{BFS}(1)$ is

$$
f_{12}=10, f_{23}=20, f_{34}=10,
$$

and

$$
x(a)=5,000, x(b)=16,000, x(c)=4,000 .
$$

The Simplex Tableau, ST(1), is 


\begin{tabular}{|c|cccccc|}
\hline$A\left(f_{i j}\right)$ & $\{a\}$ & $\{a, b\}$ & $\{a, b, c\}$ & $\{b\}$ & $\{b, c\}$ & $\{c\}$ \\
\hline$f$ & $f_{12}$ & $f_{13}$ & $f_{14}$ & $f_{23}$ & $f_{24}$ & $f_{34}$ \\
\hline$r$ & 500 & 1,500 & 2,000 & 800 & 1,800 & 400 \\
\hline$f_{12}=10$ & 1 & 1 & 1 & 0 & 0 & 0 \\
\hline$f_{23}=20$ & 0 & 1 & 1 & 1 & 1 & 0 \\
\hline$f_{34}=10$ & 0 & 0 & 1 & 0 & 1 & 1 \\
\hline 25,000 & 0 & -200 & -300 & 0 & -600 & 0 \\
\hline$x_{a}=5,000$ & 5,000 & 0 & 0 & 0 & 0 & 0 \\
\hline$x_{b}=16,000$ & 0 & 0 & 0 & 16,000 & 0 & 0 \\
\hline$x_{c}=4,000$ & 0 & 0 & 0 & 0 & 0 & 4,000 \\
\hline
\end{tabular}

Note that row 7 is the optimality test row.

The next tableau after $f_{24}$ becomes the basic variable while $f_{34}$ becomes a non-basic variable, is

\begin{tabular}{|c|cccccc|}
\hline$A\left(f_{i j}\right)$ & $\{a\}$ & $\{a, b\}$ & $\{a, b, c\}$ & $\{b\}$ & $\{b, c\}$ & $\{c\}$ \\
\hline$f$ & $f_{12}$ & $f_{13}$ & $f_{14}$ & $f_{23}$ & $f_{24}$ & $f_{34}$ \\
\hline$r$ & 500 & 1,500 & 2,000 & 800 & 1,800 & 400 \\
\hline$f_{12}=10$ & 1 & 1 & 1 & 0 & 0 & 0 \\
\hline$f_{23}=10$ & 0 & 1 & 0 & 1 & 0 & -1 \\
\hline$f_{24}=10$ & 0 & 0 & 1 & 0 & 1 & 1 \\
\hline 31,000 & 0 & -200 & 300 & 0 & 0 & 600 \\
\hline$x_{a}=5,000$ & 5,000 & 0 & 0 & 0 & 0 & 0 \\
\hline$x_{b}=17,000$ & 0 & 0 & 0 & 8,000 & 9,000 & 0 \\
\hline$x_{c}=9,000$ & 0 & 0 & 0 & 0 & 9,000 & 0 \\
\hline
\end{tabular}

Since $A\left(f_{24}\right)=\{b, c\}$ and $r\left(f_{24}\right) f_{24}=18,000$, and $f_{23}=10$, thus,

$$
\begin{aligned}
& x(a)=5,000, \\
& x(b)=800 \times 10+18,000 \times(1 / 2)=17,000, \\
& x(c)=18,000 \times(1 / 2)=9,000 .
\end{aligned}
$$

Then, the next tableau after $f_{13}$ becomes the basic variable while $f_{12}$ becomes a non-basic variable, is 


\begin{tabular}{|c|cccccc|}
\hline$A\left(f_{i j}\right)$ & $\{a\}$ & $\{a, b\}$ & $\{a, b, c\}$ & $\{b\}$ & $\{b, c\}$ & $\{c\}$ \\
\hline$f$ & $f_{12}$ & $f_{13}$ & $f_{14}$ & $f_{23}$ & $f_{24}$ & $f_{34}$ \\
\hline$r$ & 500 & 1,500 & 2,000 & 800 & 1,800 & 400 \\
\hline$f_{13}=10$ & 1 & 1 & 1 & 0 & 0 & 0 \\
\hline$f_{23}=0$ & -1 & 0 & -1 & 1 & 0 & -1 \\
\hline$f_{24}=10$ & 0 & 0 & 1 & 0 & 1 & 1 \\
\hline 33,000 & 200 & 0 & 500 & 0 & 0 & 600 \\
\hline$x_{a}=7,500$ & 0 & 7,500 & 0 & 0 & 0 & 0 \\
\hline$x_{b}=16,500$ & 0 & 7,500 & 0 & 0 & 9,000 & 0 \\
\hline$x_{c}=9,000$ & 0 & 0 & 0 & 0 & 9,000 & 0 \\
\hline
\end{tabular}

Now the numbers in the optimality test row are all nonnegative and therefore the current solution is optimal. And the corresponding revenue allocation is the following

$$
\begin{aligned}
& x(a)=7,500, \\
& x(b)=9,000+7,500=16,500, \\
& x(c)=9,000 .
\end{aligned}
$$

This is exactly as given by (18).

\section{Conclusion}

In this paper, we take an axiomatic approach to the airline alliance revenue sharing problem. We model the problem as a revenue sharing problem on a tree network, in which various paths between pairs of nodes are flights that generate revenues. Each airline owns a part of the network and the whole network is jointly shared by all airlines in the alliance. We propose and characterize a simple sharing rule that allocates the revenue of each flight equally among the airlines that are the carriers in that flight. Moreover, we show that the rule coincides with the Shapley value of the game associated with the problem. Then, we provide two extensions of the rule, allowing it to depend on the lengths or the capacities of the legs. We provide a characterization for each of these extensions.

We also consider the problem of sharing the maximum revenue of the airline alliance. To apply the duality theory in LP, we show in a lemma 
(Lemma 1) that the ILP of the problem and its LP Relaxation have the same set of solutions. Based on this result, we then show that the Owen set of the problem is nonempty. Solutions in the Owen set can be considered as solutions to the maximum revenue sharing problem. We also propose an alternative approach-the Simplex Algorithm approach. Specifically, we modify the Simplex Algorithm to implement the simple rule proposed in the beginning of the paper.

Here, we repeat our caution on the use of the Owen set as a revenue sharing solution. Since for Integer Linear Programming problems, a small change in the capacity constraints can cause a relatively large change in the value of the optimal solutions, we should avoid using dual variables if possible. On the other hand, there is no such issue in the algorithmic approach.

Finally, for future research we can include the demand constraints in the maximum revenue problem and modify the algorithm again to implement the simple or other revenue sharing rules.

\section{References}

[1] Bergantiños, G., Morento-Ternero, J. D., 2015. The axiomatic approach to the problem of sharing the revenue from museum passes. Games Econ. Behav. 89, 78-92.

[2] Bergantiños, G., Vidal-Puga, J., 2007. A fair rule in minimum cost spanning tree problems. J. Econ. Theory 137, 326-352.

[3] Bogomolnaia, A., Holzman, R., and Moulin, H., 2010. Sharing the cost of a capacity network. Math. Oper. Res. 35, 173-192.

[4] Dantzig, G. B., 1963. Linear Programming and Extensions. Princeton University Press.

[5] de Boer, S. V., Freling, R., and Piersma, N., 2002. Mathematical programming for network revenue management revisited. Eur. J. Oper Res. $137,72-92$.

[6] Garfinkel, R. S., Nembauser, G. L., 1972. Integer Programming. John Wiley \& Sons, New York. 
[7] Ginsburgh, V., Zang, I., 2003. The museum pass game and its value. Games Econ. Behav. 43, 322-325.

[8] Kimms, A., Çetiner, D., 2012. Approximate nucleolus-based revenue sharing in airline alliances. Eur J. Operat. Res 220, 510-521.

[9] Littlechild, S., Owen, G., 1973. A Simple Expression for the Shapley Value in a Special Case. Management Sci. 20, 370-372.

[10] Morrison, S., Winston, C., 1986. The economic effects of airline deregulation. The Brookings Instition, Washington DC.

[11] Moulin, H., 2002. Axiomatic cost and surplus sharing. In: Arrow, K.J., Sen, A.K., and Suzumura, K. (Eds.), Handbook of Social Choice and Welfare. North-Holland, Amsterdam.

[12] Moulin, H., Laigret, F., 2011. Equal-need sharing of a network under connectivity constriants. Games Econ. Behav. 72, 314-320.

[13] Netessine, S., Shumsky, R., 2005. Revenue management games: Horizontal and vertical competition. Management Sci. 51, 813-831.

[14] Ni, D., Wang, Y., 2007. Sharing a polluted river. Games Econ. Behav. 60, 176-186.

[15] Owen, G., 1975. On the core of linear production games. Math. Prog. 9, 358-370.

[16] Shapley, L. S., 1953. A Value for n-Person Games. In: H. W. Kuhn and A. W. Tucker (Eds.), Contributions to the Theory of Games II, Annals of Mathematics Studies 28, 307-17.

[17] Shapley, L. S., 1971. Cores of Convex Games. Int. J. Game Theory 1, 11-26.

[18] Sharkey, W., 1995. Network models in economics. In: Ball, M.O., Magnanti, T.L., Monma, C.L., Nemhauser, G.L. (Eds), Network Routing, Handbooks in Operations Research and Management Science (8). NorthHolland, Amsterdam. 
[19] Talluri, K. T., van Ryzin, G. J., 2004. The Theory and Practice of Revenue Management. Springer, New York.

[20] Hu, X., Caldentey, R., and Vulcano, G., 2012. Revenue Sharing in Airline Alliances. Management Sci. 59, No. 5, 1177-1195. 